\title{
Caracterización de la exposición a polvo orgánico en el área de producción de alimento balanceado y granjas avícolas en la empresa "Megaves Cía. Ltda."
}

\section{Organic dust exposure characterization in the area of balanced diet production and poultry farms of the company "Megaves Cía Ltda."}

\author{
Luis Rojas Viteri ${ }^{1}$, Amalia García Prieto ${ }^{1}$
}

${ }^{\prime}$ Universidad Tecnológica Equinoccial, Quito, Ecuador

\begin{abstract}
Resumen
El presente estudio definió la exposición de los trabajadores del área de alimento balanceado y producción avícola de la empresa Megaves a polvo orgánico. El estudio fue descriptivo de corte transversal, donde se comparó la exposición a polvo orgánico total (fracción respirable) con los límites permisibles del Instituto Nacional de Seguridad e Higiene en el Trabajo (INSHT) de España. Una encuesta higiénica fue aplicada para la caracterización de los determinantes de riesgo. Como instrumento de medición de polvo para caracterizar la exposición se utilizó el equipo EVM3 configurado a un flujo de 1.7 1/minuto, y con una captación activa de polvo con tamaño de partícula inferior a 10 micras, considerando el $75 \%$ de la jornada laboral. Las muestras obtenidas fueron pesadas de acuerdo a la norma técnica MTA/MA-014/A88 del INSHT "Determinación de materia particulada (total y fracción respirable) en aire - Método gravimétrico". Los resultados de las mediciones de polvo estuvieron por debajo del límite permisible de $4 \mathrm{mg} / \mathrm{m}^{3}$, por lo que se concluyó que la exposición a este material particulado no constituye un riesgo para la salud de los trabajadores en la empresa objeto de estudio.
\end{abstract}

Palabras clave: exposición, orgánico, polvo, prevención, avícola.

\begin{abstract}
This study defined exposure to organic dust of workers in the area of balanced diets production and poultry farms of the company Megaves. This was a descriptive and cross-sectional study, in which the exposure to total organic dust (inhalable fraction) was compared to the permissible limits of Instituto Nacional de Seguridad e Higiene en el Trabajo (INSHT) from Spain. A hygienic survey was applied with the aim of characterizing the risk determinants. EVM3 was used to measure dust and to characterize the exposure. It was configured to a flow of $1.7 \mathrm{l} /$ minute, and with an active uptake to dust with particle size lower than 10 microns. The measurements were developed for the $75 \%$ of the work day. The samples were weigthed according to the technical standard of the INSHT MTA/MA-014/A88 "determination of matter (total and inhalable fraction) particulate in air - gravimetric method". The dust concentrations found were below the permissible limit of $4 \mathrm{mg} / \mathrm{m}^{3}$, so that the exposure to particulate matter is not a real risk to the health of workers in the company under study.
\end{abstract}

Keywords: dust, exposure, organic, poultry, prevention 


\section{Introducción}

La avicultura es una de las principales actividades agropecuarias en Ecuador. Las aves de corral, específicamente los "pollos de engorde", se caracterizan por su rápida reproducción, crecimiento, siendo su precio menor que el de otras especies. Los pequeños inversionistas desarrollan la actividad avícola de manera informal sin tener conocimiento de la legislación ecuatoriana. Sin embargo, el mediano y gran inversionista se asocian teniendo beneficios mutuos, reduciendo costos y aumentando la productividad (Chang et al., 2009). La evolución del consumo per cápita de carne de pollo en Ecuador, detallado en la Figura 1. Es notorio y significativo el incremento del consumo de 1990 hasta la actualidad, lo que evidencia el potencial de crecimiento de esta actividad en el futuro y demuestra la importancia que este producto ha llegado a tener dentro de las preferencias de los consumidores ecuatorianos, (Orellana, 2007). El sector avícola constituye una de las principales fuentes de empleo en el Ecuador, no solo en forma directa sino también indirecta (cultivo de maíz, elaboración de balanceados, distribución y venta de productos finales e intermediarios) (Orellana, 2007). En la actualidad, en el país existen aproximadamente 1,567 planteles avícolas que generan más de 25,000 empleos directos.

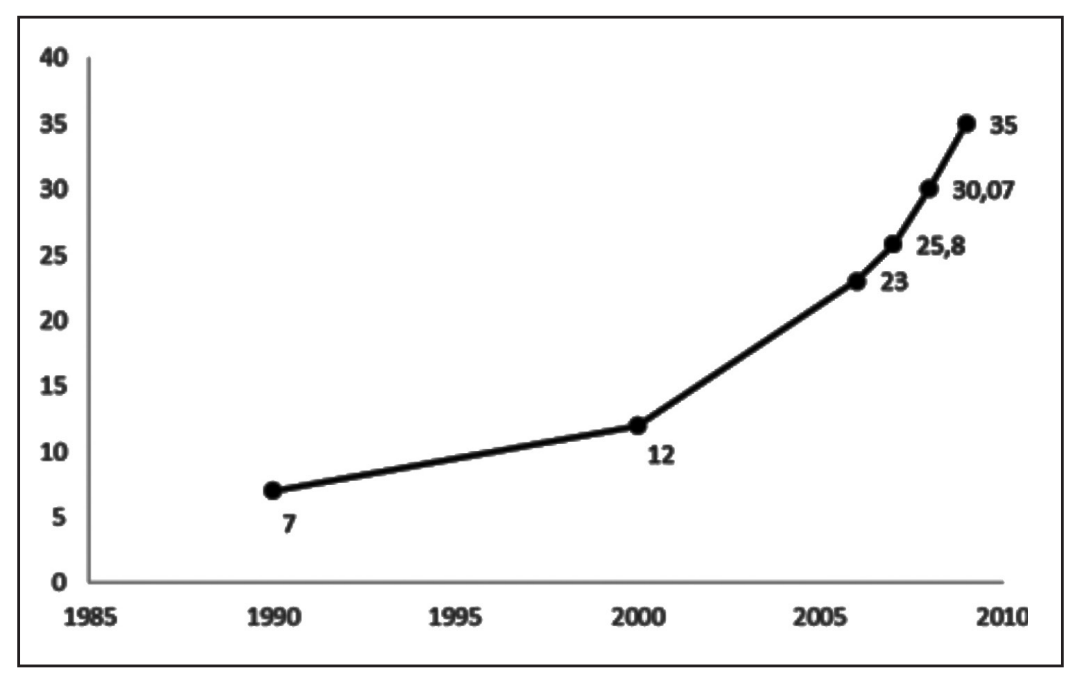

Figura 1. Evolución del consumo per cápita de pollo en Ecuador (kg), CONAVE (2007).

La proyección de producción de pollo de CONAVE para el 2013 fue de 230 millones de pollos de engorde, con un consumo per cápita de pollo de 35 $\mathrm{kg} / \mathrm{año} /$ habitante. Cabe mencionar que la producción avícola nacional en el Ecuador abastece el ciento por ciento de la demanda de carne de pollo y de huevos de consumo y alrededor del $95 \%$ de la demanda de carne de pavo (CONAVE, 2013).

Megaves Cía. Ltda. se dedica a la producción, procesamiento y comercialización de pollos broiler. $\mathrm{Su}$ estructura organizacional está constituida por cuatro áreas en el proceso productivo: producción de balanceados, crianza de pollos, procesamiento y comercialización. Este estudio se enfoca en las dos primeras fases del proceso.

Según Lenhart (2001), las condiciones de trabajo de los galponeros han mejorado con el desarro- llo de la industria avícola. Sin embargo, a pesar del esfuerzo de los avicultores por mejorar dichas condiciones, la crianza de las aves obliga al galponero a trabajar siete días a la semana. En consecuencia, la exposición al polvo orgánico se produce durante varios días consecutivos. Durante la elaboración de alimento balanceado, así como en las labores diarias del cuidado de las aves se produce polvo orgánico ambiental a partir del alimento balanceado, heces fecales, plumas, entre otros. La inhalación del mismo puede causar lesiones severas dentro del aparato respiratorio. Los efectos crónicos a largo plazo de la exposición al polvo pueden incluir congestión pulmonar, bronquitis crónica y neumonía, y diferentes sensibilidades y alergias al polvo. La exposición crónica al polvo puede causar problemas respiratorios graves como asma, enfisema y neumonitis hipersensible (pulmón de granjero). Estas enfermedades pueden requerir medicamentos y cuidados médicos con- 
tinuos (State Compensation Insurance Fund, 2010). Es preciso determinar la concentración de polvo total en las áreas de trabajo de la compañía avícola objeto de estudio con el fin de compararla con los límites permisibles establecidos para este tipo de partículas y garantizar el estado de salud de los trabajadores. Adicionalmente, es necesario determinar el tiempo de exposición de los trabajadores al material particulado, además de identificar si poseen el equipo de protección adecuado para realizar su actividad. Según Lenhart (2001) no todas las mascarillas proporcionan el mismo nivel de protección, y el tipo elegido para un corral de confinamiento puede variar de acuerdo a la concentración y tipo de polvo que exista en el área. Los niveles de polvo en el aire son máximos durante las operaciones de atrapamiento de pollos, a veces hasta el punto de que no se alcanza a ver de un extremo del corral al otro.

Hasta el año 2014 la empresa Megaves no había realizado mediciones de la concentración de polvo orgánico a la que están expuestos los trabajadores y las medidas preventivas que se han tomado; como la utilización del equipo de protección personal, no garantizan que la exposición a dichos polvos orgánicos no sea nociva para la salud de los trabajadores. Según Lenhart (2001), los trabajadores avícolas que no llevan protección respiratoria adecuada y se encuentren expuestos a polvo orgánico, corren el riesgo de padecer enfermedades respiratorias como rinitis alérgica, bronquitis, asma, neumonitis por hipersensibilidad o alveolitis alérgicas y síndrome tóxico por polvo orgánico. Los trabajadores avícolas generalmente presentan síntomas respiratorios agudos y crónicos como tos, sibilancias, excesiva secreción de moco, disnea y dolor y sensación de opresión en el pecho.

En el presente trabajo se propuso caracterizar la exposición al polvo orgánico generado en el ambiente de trabajo y los determinantes de exposición a este riesgo, además de promover medidas preventivas y correctivas necesarias para preservar la seguridad y salud de los trabajadores del sector avícola. La investigación desarrollada fue descriptiva y de corte transversal, y se basó en literatura previa (Arias y Bértona, 2012; Díaz et al., 1998; Díaz et al., 2011; Martí, 2009).

\section{Materiales y métodos}

La caracterización de los determinantes de riesgo (condiciones de trabajo, organización del trabajo y ambiente de trabajo) se realizó a través de la aplicación de una encuesta higiénica que fue previamente validada por tres expertos y probada durante una prueba piloto. La encuesta se aplicó a los 19 trabajadores de la empresa.

La observación directa fue la técnica empleada para describir el proceso productivo e identificar la maquinaria, equipos y fuentes de producción de polvo, haciendo uso de una ficha de observación. La exposición a polvo orgánico se determinó en tres áreas de actividad: producción de balanceado (a cinco trabajadores), producción de pollo-granjas avícolas (en cinco galponeros) y recolección de pollo (a nueve recolectores). Para la medición de polvo en las áreas de trabajo seleccionadas se utilizó el equipo EVM3 que fue programado para recolectar el polvo total (tamaño de partícula inferior a $10 \mu \mathrm{m}$ ), que contempla la fracción respirable, y se estableció un caudal para la captación activa de $1.7 \mathrm{l} / \mathrm{m}^{3}$.

La recolección de muestras se realizó considerando el $75 \%$ de la jornada laboral. Se tomaron tres muestras de aire de un galpón seleccionado (muestras 2,3 y 4) y tres muestras en la etapa de recolección de pollo, que es la etapa de mayor generación de polvo orgánico (muestras 1, 7 y 9). Por otra parte, en el área de producción de alimento balanceado se tomaron tres muestras de polvo total (muestras 5, 6 y 8). En la Tabla 1 se muestran los tiempos de muestreo en los diferentes puntos de muestreo.

Se siguió el protocolo establecido en la norma técnica MTA/MA-014/A88 del INSHT “determinación de materia particulada (total y fracción respirable) en aire - método gravimétrico". Las muestras recogidas se trasladaron al laboratorio donde fueron pesadas en una balanza analítica. Los instrumentos empleados fueron calibrados previamente.

De acuerdo a dicha norma, la concentración de polvo total (C) se halla considerando por un lado el peso de material particulado retenido $(\mathrm{P})$, que resulta de la diferencia entre el peso de la muestra $\left(\mathrm{P}_{2}\right.$ : cassette más polvo retenido, en $\mathrm{mg}$ ) y el peso del cassette $\mathrm{P}_{1}$ en $\mathrm{mg}$, y por otro, el volumen de aire muestreado $(\mathrm{V})$ en $\mathrm{m}^{3}$.

La expresión utilizada es la siguiente:

$$
\mathrm{C}\left(\mathrm{mg} / \mathrm{m}^{3}\right)=\mathrm{P} / \mathrm{V}
$$


El nivel de referencia adoptado fue el valor límite ambiental-exposición diaria (VLA-ED) y per- misible para polvo, granos (avena, trigo, cebada) según el INSHT, el cual corresponde a 4 ppm.

Tabla 1. Tiempo de muestreo

\begin{tabular}{cccc}
\hline Muestra & $\begin{array}{c}\text { Tiempo } \\
\text { (minutos) }\end{array}$ & Muestra & $\begin{array}{c}\text { Tiempo } \\
\text { (minutos) }\end{array}$ \\
\hline 1 & 30 & 6 & 271 \\
2 & 361 & 7 & 20 \\
3 & 365 & 8 & 360 \\
4 & 360 & 9 & 60 \\
5 & 361 & & \\
\hline
\end{tabular}

\section{Resultados y discusión}

\subsection{Procesos en la actividad avícola}

\subsubsection{Producción de alimento balanceado}

La producción de alimento balanceado consiste en una cadena de elaboración de raciones alimenticias con base en mezclas de materia prima en cantidades necesarias para cada etapa de crecimiento de las aves. Existen diferentes tipos de alimento (broiler 1-4) en función de la edad del pollo de engorde. Las materias primas empleadas se clasifican en micro ingredientes (núcleos, sal común, bicarbonato de sodio, anticoccidiales ionóforos, enzimas y promotores del crecimiento), que se incluyen en el alimento en cantidades que oscilan entre 200 y 8,000 g) y los macro ingredientes (entre 8 y $60 \mathrm{~kg}$ ). El maíz y pasta de soya son macro ingredientes que se reciben a granel. Estos se descargan desde un camión. El maíz se lleva por un elevador hacia la zona de limpieza de grano mientras que la pasta de soya va a una tolva de espera para el pesaje. El grano de maíz se separa en maíz entero, partido y fino mediante el uso de mallas.

El entero se dirige a la tolva de espera para pesaje mientras que los otros dos se depositan en otro lugar para reutilizarlo con otros fines. Los ingredientes pasan desde la tolva de espera a la de pesaje donde se pesan en una balanza electrónica que está incorporada en la tolva y luego se almacenan en silos. Hay otros macro ingredientes que se receptan en sacos como son el grano de soya, la harina de pescado, la caliza y el fosfato. Para la fabricación del balanceado también se utilizan grasa animal y aceite vegetal.

A través de transportadores horizontales los ingredientes pasan de los silos a una tolva de espera. El maíz, pasta de soya y grano de soya pasan a un molino de martillos donde se reduce su tamaño para luego, junto a los micro ingredientes, llegar a la tolva de mezclado donde se mezclan todos los ingredientes uniformemente para producir el balanceado. Finalmente se envasa en sacos de polipropileno (de $45.36 \mathrm{~kg}$ cada uno) que se usarán en los comederos de los galpones.

\subsubsection{Proceso de producción avícola}

El proceso de producción avícola se desarro1la en un galpón e implica todas las actividades del cuidado del pollo de engorde para obtener finalmente carne animal. Antes de la recepción del "pollito" hay un acondicionamiento previo como es la colocación de bandejas de cartón desechables. También se coloca antibiótico en el agua de los bebederos y se precalienta el galpón hasta alcanzar una temperatura de $25^{\circ} \mathrm{C}$. El "pollito" se recibe en la zona de crianza del galpón y un indicador de que la temperatura es óptima es que su distribución es uniforme. La ventilación del galpón se controla a través de las cortinas exteriores e interiores.

A partir de la segunda semana se hacen otras actividades como la limpieza y regulación del sistema de bebederos de niple; el reemplazo de las bandejas de cartón por los comederos automáticos (a los 15-18 días de vida del pollo); el ingreso de alimento a la granja; fumigaciones alrededor de los galpones; recolección de aves muertas y otras actividades que son continuas durante el ciclo de producción como la medición del cloro y del pH en el agua del galpón.

\subsection{Fuentes de emisión de partículas}

En el área de producción de alimento balanceado se identificaron los procesos críticos de gene- 
ración de polvo. Las tolvas de recepción de maíz y soya no son totalmente herméticas lo que produce la salida de polvo al ambiente de trabajo. En el proceso de mezclado los trabajadores introducen manualmente los micro ingredientes, lo cual genera una gran cantidad de polvo. También se produce polvo en el proceso de molienda.

En el área de producción de pollo las actividades que más polvo generan son la alimentación de las aves y el barrido de las camas en el galpón, mientras que en la recolección de animales para llevarlos a la planta de faenamiento, los trabajadores sujetan al pollo por las patas, causando estrés a los animales, los cuales aletean y corren, generando una gran cantidad de polvo en el galpón.

\subsection{Aplicación de la encuesta higiénica}

La encuesta se aplicó a 19 trabajadores, 18 de los cuales fueron hombres. El 47.37\% trabajan en el área de recolección de pollo mientras que el $26.32 \%$ en la producción de balanceado y $26.32 \%$ en la producción de pollo. El 73.68\% de los informantes dice trabajar en locales cerrados. Según los encuestados los factores de riesgo a los que consideran se encuentran más expuestos son humedad y polvillo con un $94.74 \%$, frío (73.68\%) y viento $(63.16 \%)$.

Respecto a la organización del trabajo, aproximadamente la mitad de los encuestados considera que sí hay especialización del trabajo mientras que un $36.84 \%$ respondió que existe a veces. En la empresa todos los trabajadores están afiliados al
Instituto Ecuatoriano de Seguridad Social (IESS), existe un sistema de pagos puntual y el $36.84 \%$ del personal tiene rotación entre las diferentes áreas.

En cuanto a las condiciones de trabajo, el $94.74 \%$ de los informantes realiza horas extras. La totalidad de trabajadores manifestó que la empresa proporciona equipos de protección personal y que los utiliza, y el $94.74 \%$ apuntó que se reponen cuando termina su vida útil o se deterioran y que han recibido capacitación sobre seguridad y prevención de riesgos del trabajo. Con respecto a los sistemas de protección colectiva, el $68.42 \%$ informó de que no cuentan con dichos sistemas en las áreas de trabajo.

El $94.74 \%$ de los trabajadores encuestados afirmó estar informado sobre los posibles riesgos en su puesto de trabajo y tener conocimiento de cómo actuar para prevenir dichos riesgos en su puesto de trabajo. El 100\% respondió que sí hay servicio médico en la empresa, y que solo a un $36.84 \%$ le han realizado exámenes médicos antes de entrar en la empresa. Sin embargo al $100 \%$ se le realiza exámenes periódicos.

\subsection{Determinación de concentración de polvo orgánico}

La aplicación del método gravimétrico en los puntos de muestreo seleccionados permitió determinar la concentración de polvo $(\mathrm{C})$ en diferentes áreas de trabajo. En la Tabla 2 se muestran los resultados obtenidos, además del promedio de temperatura $\left(\mathrm{T}^{\circ}\right)$ y humedad $(\mathrm{H})$ hallados en cada medición. Las concentraciones de polvo halladas en las áreas de trabajo estuvieron por debajo del límite permisible adoptado de $4 \mathrm{mg} / \mathrm{m}^{3}$.

Tabla 2. Concentración de polvo.

\begin{tabular}{ccccc}
\hline o & Área & Concentración $\left(\mathbf{m g} / \mathbf{m}^{3}\right)$ & H $(\mathbf{\%})$ & Temperatura $\left({ }^{\circ} \mathbf{C}\right)$ \\
\hline $\mathbf{1}$ & Recolectores & 0.26 & 75 & 13.0 \\
$\mathbf{2}$ & Galpones & 0.24 & 66,4 & 19.5 \\
$\mathbf{3}$ & Galpones & 0.26 & 57.0 & 23.0 \\
$\mathbf{4}$ & Galpones & 0.07 & 63.0 & 19.8 \\
$\mathbf{5}$ & Planta de alimentos & 0,26 & 43,4 & 24.4 \\
$\mathbf{6}$ & Planta de alimentos & 0.20 & 48,4 & 22.6 \\
$\mathbf{7}$ & Recolectores & 0.54 & 73,2 & 17.8 \\
$\mathbf{8}$ & Planta de alimentos & 0.03 & 48,4 & 22.6 \\
$\mathbf{9}$ & Recolectores & 0.28 & 60.0 & 18.1 \\
\hline
\end{tabular}


El área de recolección es el espacio donde se determinó la mayor concentración de polvo, sin embargo, el tiempo que destinan los trabajadores a esta actividad es menor que en las otras áreas.

Estos resultados permiten afirmar que el personal de la empresa está expuesto a una cantidad tolerable de polvo total (fracción respirable), disminuyendo la posibilidad de que puedan contraer, a causa del trabajo, patologías de vías respiratorias bajas. La temperatura tiene una relación inversamente proporcional con la humedad relativa, y esto se puede evidenciar en las mediciones realizadas en el estudio en cada una de las áreas (Flores Cerdan, 1998).

\section{Conclusiones}

El aporte del presente estudio sirve de precedente para nuevas investigaciones que pretendan encontrar resultados concluyentes con respecto al tamaño de las partículas de polvo que se evidencian en el sector avícola, ya que el alcance de este proyecto fue definir si existe la presencia de polvo total (fracción respirable) que pueda afectar la salud de los trabajadores.

Los trabajadores revelaron que visualizan una gran cantidad de polvo en las áreas de la empresa, sin embargo, de acuerdo a los resultados de las me-

\section{Referencias}

Arias, M. C., Bértona, G. (2012). Caracterización de la exposición a polvo de sílice y a ruido en los puestos de trabajo del sector de la pizarra de la provincia de Lugo.

CONAVE (2013). Estadísticas avícolas del Comité Nacional de Vigilancia Epidemiológica. Corporación Nacional de Avicultores del Ecuador.

Chang, S., Verdezoto, A., Estrada, L. (2009). Análisis de la avicultura ecuatoriana, Tesis, Escuela Politécnica del Litoral, Guayaquil, Ecuador.

Díaz, H., González, R. M., Novas, A. J., Linares, T. M., (2011). Caracterización de la exposición a polvo. Premisa para el abordaje integral en los servicios de salud. Revista cubana de salud y trabajo, 12 (3): 1-5. diciones realizadas, la concentración de polvo total está por debajo del límite permisible establecido. Por tanto, los trabajadores no se exponen al riesgo de sufrir patologías asociadas a las vías respiratorias bajas como epoc, pulmón del granjero, entre otros.

Sería recomendable desarrollar un estudio de la fracción de material particulado con diámetro superior a las diez micras (polvo visible) ya que los trabajadores podrían contraer patologías de las vías respiratorias altas, como alergias, rinitis alérgica, y otras patologías como problemas cutáneos, pterigion, entre otras. En el presente trabajo se identificó que los trabajadores laboran una media de nueve horas diarias siendo este un factor importante puesto que la exposición se alarga. Sin embargo, en el área de recolección de pollo, donde se determinó una mayor concentración de polvo, el tiempo de exposición es menor, oscila entre 1 - 3 horas.

Las áreas de la empresa Megaves no cuentan con sistemas de protección colectiva, sin embargo la empresa brinda equipo de protección respiratoria para salvaguardar la salud de los colaboradores. La legislación ecuatoriana vigente establece que las empresas deben realizar un control de riesgos primordialmente sobre la fuente de origen por lo que es recomendable diseñar sistemas de protección colectiva en las distintas áreas.

Díaz, H., Ibarra E. J. Perdomo, M., Duarte, O. (1998), Evaluación de la exposición al polvo en puestos de trabajo de contingentes de la construcción. Instituto de Medicina del Trabajo. Revista cubana higiene y epidemiologia; 36 (2),147-51.

MTA/MA-014/A11 (2012). Determinación de materia particulada (fracciones inhalable, torácica y respirable) en aire - Método gravimétrico. Revisión actualización del MTA/MA-014/A88, España.

Flores Cerdan, M. (1998) Humedad relativa y salud, recomendaciones para la prevención y control de la legionelosis. Capítulo 8 - Humectadores. Ministerio de Sanidad, Servicios Sociales e Igualdad, España. 
Instituto Nacional de Seguridad e Higiene en el trabajo INSHT (2007). Centro Nacional de Verificación de Maquinaria. Vizcaya, España

Lantarka, S. L. (2012). Límite de exposición profesional para agentes químicos en España. Ministerio de Empleo y Seguridad Social, Instituto Nacional de Seguridad e Higiene en el Trabajo, Madrid, España.

Lenhart, S. W. (2001). Producción de aves de corral y de huevos. Enciclopedia de salud y seguridad en el trabajo.

Martí, A. (2009) Exposición laboral a polvo inhalable en plantas de compostaje de residuos sólidos urbanos. INSHT. Centro Nacional de Condiciones de Trabajo. Barcelona, España.
Orellana J. (2007). El gremio avícola nacional sus acciones, incidencias de las mismas y la necesidad del fortalecimiento gremial. Informe CONAVE - Corporación Nacional de Avicultores del Ecuador. Quito, Ecuador.

State Compensation Insurance Fund (2010). Polvo agrícola página oficial de California (State Fund). California, USA. 